\title{
IMMEDIATE IMPLANT PLACEMENT WITH PROVISIONALIZATION USING AUTOGENOUS BONE GRAFT PARTICULATES VS DEPROTEINIZED BOVINE BONE VS NANO-HYDROXYAPATITE AS A SPACE FILLING MATERIAL IN THE MAXILLARY ESTHETIC ZONE
}

\author{
Mohamed Tarek Youssef Omar*, Amr Hosny El Khadem ${ }^{* * *}$ and Mohamed Atef Abdel Rassoul***
}

\begin{abstract}
Background: Latest dentistry had been reported as fast and growing evolution. Regarding the implant- rehabilitation protocols, they had been altered in order to fulfill patient's increasing prospects in terms of ease, appealing and smaller treatment period. Aim; evaluate bone and soft tissue changes after tooth extraction and immediate implant insertion with immediate provisionalization in the anterior maxilla,
\end{abstract}

Methods: Thirty immediate implants were placed in anterior maxilla, a novel synthetic bone graft (Geistlich Bio-Oss ${ }^{\circledR}$ ), nano bone (Nano streams) was used to augment the gap between the implant and the alveolar bone interface. Implants diameter used in this study is $(3.7 * 14)$ compared to the size of the socket were used. A fixed screw retained provisional restoration was delivered immediately after implant placement. Cone beam computed tomography (CBCT) scans were taken to evaluate bone changes immediate after surgery and after 6-months follow up. To assess soft tissue changes, clinical vertical measurements were performed by using pink esthetic score.

Results: Mean horizontal bone loss for Group I, II and III were $(0.671),(0.380)$ and $(0.289)$ $\mathrm{mm}$ respectively. This was statistically not significant as $\mathrm{p}=0.057$. Mean vertical bone loss for Group I, II and III were $(0.212),(0.233)$ and $(0.262) \mathrm{mm}$ respectively. This was statistically not significant as $\mathrm{p}=0.807$.

Conclusion: Immediate implant with immediate temporization using graft materials to augment jumping gap is a successful treatment modality for rehabilitation of hopeless teeth in the esthetic zone. The type of graft materials doesn't significantly affect on the outcome (horizontal and vertical bone loss) following implant installation with immediate temporization in the esthetic zone.

KEYWORDS: Implant, Immediate, Graft, Esthetic, Maxilla

\footnotetext{
* Implantology. Dentistry. Cairo University

** Assistant Professor, Oral and Dental Removable Prosthodontics, Dentistry. Cairo University

*** Lecturere, Oral and Maxillofacial Surgery, Dentistry. Cairo University
} 


\section{INTRODUCTION}

The word 'Osseo integration' was first introduced by Branemark in 1952. Osseo integration is defined as direct connection in implant and live bone cells interface. Early, the original protocol for fitting of the dental implants was 6-8 months' post extraction. After installation of the implant, healing period for Osseo integration time was six months for maxilla and three months for the mandible. In 1990s, implant insertion was mostly operated in $100 \%$ healed bone tissue. Today, this technique has lost its priority because of the introduction of the implant shape and surface characteristics. Different researches investigate that immediate implantation has a 90 $100 \%$ success in survival rate. However, primary stability of the implant at the recent extraction socket still has high advantage. Particularly after extraction of single root teeth, implant insertion into the recent extraction socket by filling the gap with graft materials was clearly investigated ${ }^{(1)}$.

Immediate implant insertion had more benefits in relation to delayed implant insertion, which implants in recent extraction sites could be placed in the same anatomical conditions thereby minimizing the use of angled abutments, Osseo integration was more beneficial, the bony receptors were preserved by preventing atrophy of the alveolar ridge thereby preventing recession of the soft tissues, immediate placement of implants eliminates contaminants out of extraction socket, healing periods for primary healing of the soft tissues, and regeneration of the bony structure are eliminated,immediate restorations could be provided for better appearance. ${ }^{(2)}$

This gap at the implant and surrounding bone interface was called the gap or jumping distance. The gap consisted of two measurements: Horizontal defect width and vertical defect height. The term "jumping distance" referred to the ability of bone to connect the horizontal gap and fill the space. The main outcome of immediate implant insertion was to provide an Osseo integrated fixture needed for an aesthetic and functional re-establishment. Bone occupy in the gap in the implant and the peripheral bone interface was important. The buccal side of implant was of great concern, especially in the aesthetic zone, because the buccal bony plate is usually thin and its resorption could lead to soft tissue recession. The outcome of the surgical management of the buccal gap was perfect bone fill in the space, most coronal level of bone-to-implant contact and the least amount of buccal bone loss and soft- tissue recession. ${ }^{(3)}$.

In listed case report, it was discovered that it is possible for a large gap to fill with integrated bone without any management of the gap by placement immediate implant placement with $4.2 \mathrm{~mm}$ horizontal gap to the facial plate and allow to heal without any management techniques. The fixture was removed for histology 10 months following implant placement along with some facial bone. The histology result showed Osseo integration of implant which serving to confirm that the gap had filled without any bone graft or barrier membrane ${ }^{(4)}$.

It was evaluated the success of immediate endosseous implants inserted with autogenous bone graft to fill the peri-implant space. Thirtytwo implants were inserted in eight beagle dogs. The right and left lateral incisors in the maxilla and the mandible of all animals were removed, and immediate post extraction implants were placed. In the control sites, no bone grafts or barrier membranes were used. He listed that the filling of the peri-implant bone defects with autogenous bone grafts showed a better outcome compared to unfilled defects ${ }^{(5)}$.

It was examined the five year hard and soft tissue changes at implants placed in the anterior maxilla. Thirty-seven micro threaded implants were placed in twenty-one patients into extraction sockets with and without facial bone deficits by a flapless approach. He listed that interproximal marginal bone levels, survival rates, and esthetic results remain fixed 
at the five-year healing periods in implants used in an immediate insertion, reconstruction, and provisionalization concept. Facial marginal bone levels reduced slightly; however, this reduction did not affect the PES so far. The most common used type is the deproteinized animal bone mineral which commercially known as Bio-oss, which is composed of natural bone minerals without organic element Xenografts ${ }^{(6)}$.

Hydroxyapatite (HA) nano particles had been greatly introduced as a bio safe bone graft, especially for contact with bone tissue, had similarity to mineral bone and introduced a carbonated and partially substituted apatite, based on nanocrystal aggregates, and associated with collagen, building up 3-D structures present in various bone tissue conformations like trabecular or cancellous bone. ${ }^{(7)}$.

It was evaluated histologically the efficacy of micro-, nano-, or mixed-composite of hydroxyapatite (HA) graft in treatment of postsurgical gaps around dental implants in mongrel dogs. Immediate implant was used post extraction of the lower third premolar in mongrel male dogs. Critical-size defects were created in in one-piece proximal alveolar bone to each implant. Defects were divided randomly into four groups of two animals based on biomaterials used for treatment: 1) received no treatment (negative control); 2) defects treated with nano-HA bone graft; 3) defects treated with micro-HA bone graft; and 4) defects treated with a mixed composite of micro-HA and nano-HA. Animals were sacrificed at two months and histological evaluation was performed. She concluded that nano-hydroxyapatite bone graft introduced better Osseous formative conditions than micro-HA or mixed-HA bone graft in new bone formation in standardized surgically created defects around dental implants ${ }^{(8)}$.

It was studied that implant surgery protocols and the high attention on aesthetics have introduced a change in treatment results from implant survival to success in aesthetic zone. The aim of this retrospective study was to evaluate the results of immediate single tooth implant insertion and provisionalization in the aesthetic zone regarding hard and soft peri- implant tissue parameters and patient's related results measures. He concluded that placement of implants into fresh extraction sites followed by immediate provisionalization resulted in a high survival, minimum peri-implant bone loss, good aesthetics and satisfied patients after a mean follow-up period of four years ${ }^{(9)}$.

It was evaluated that long term (2-5 years) tissue changes around immediately placed and restored implants in the anterior maxilla using flapless surgery and immediate hard tissue augmentation. He put thirty implants in thirteen patients, with same immediate implant placement protocols with immediate temporization and augmented with deproteinized particulate xenograft. Healing period of osseointegration up to period of 2-5 years and they found complete soft tissue around implant is healthy and advantageous ${ }^{(10)}$.

It was assessed that effect of altering the field of view on the dimensional accuracy of CBCT imaging had a great role. The implant-bone models were randomly numbered from 1 to 13 by the principal investigator. CBCT examinations of each bone model were performed using Cranex3Dx CBCT (Helsinki, Finland) machine. Each model was scanned four times with standardized tube current and voltage of $12.5 \mathrm{~mA}$ and $90 \mathrm{kVp}$ respectively at four different FOVs. The FOVs used were as following: Small FOV: 50 x $50 \mathrm{~mm}$ with voxel size $200 \mu \mathrm{m}$, Midi FOV: $61 \times 78 \mathrm{~mm}$ with voxel size $300 \mu \mathrm{m}$, Medium FOV: 78 x $78 \mathrm{~mm}$ with voxel size $300 \mu \mathrm{m}$, Large FOV: 78 x $150 \mathrm{~mm}$ with voxel size $350 \mu \mathrm{m}$. Increasing the FOV size together with voxel size could undesirably affect the accuracy of CBCT linear measurements, especially when small distances are to be evaluated ${ }^{(11)}$. 


\section{METHODS AND METHODS}

A total of thirty extractions sockets in the esthetic zone indicated for implant placement, excluding female patients to avoid hormonal imbalance mishaps, were selected from the outpatient clinic of Oral Implantology Department, Faculty of Dentistry, Cairo University.

Thirty extraction sockets were randomly divided into three groups using block randomization with stratification (block size 4). using a formula on Microsoft excel software.

Based on a previous study by Koutouzis \& Lundgren, 2010, the difference in Crestal Bone Level Changes between at least 2 groups is $0.5 \pm 0.33 \mathrm{~mm}$. Using power $80 \%$ and $5 \%$ significance level we will need to study 8 in each group. This number is to be increased to a sample size of 10 in each group to compensate for losses during follow up. Sample size calculation was achieved using PS: Power and Sample Size Calculation software Version 3.1.2 (Vanderbilt University, Nashville, Tennessee, USA.

The first group (Autogenous group); included 10 extraction sockets in patients which received immediate post extraction implant placement with autogenous bone graft to augment the gap between the residual labial bone and implant surface with immediate temporization.

The second group (Xenograft group); included 10 extraction sockets in patients which received immediate post extraction implant placement with xenograft to augment the gap between the residual labial bone and implant surface with immediate temporization.

The third group (Nano-hydroxyapatite group); included 10 extraction sockets in patients which received immediate post extraction implant placement with nano-hydroxyapatite alloplast to augment the gap between the residual labial bone and implant surface with immediate temporization.
First radiographic examination; Radiographic examination included preoperative digital panoramic radiograph with 1:1 magnification was taken for a candidate that had multiple teeth as a primary survey to obtain an approximation of the available bone height and the presence of periapical pathosis. Also Radiographic periapical was taken for patient that had single tooth as preoperative screening

Second radiographic examination; Preoperative Cone Beam Computed Tomography CBCT scan for the selected patients after plain radiograph screening has been made to accurately measure the dimensions of ridge. Blue sky bio software was used for implant planning.

A scout view was obtained, and adjustments were made to ensure that the all patients were correctly aligned in the scanner according to the adjustment of light beam before acquisition. The machine is supplied with Amorphous Silicon Flat Panel Sensor with Cesium Iodide (CsI) scintillator, $0.5 \mathrm{~mm}$ focal spot size, 14-Bit grey scale resolution.

The following protocol was used for all the scans of the study: Tube voltage: $120 \mathrm{kVp}$, Milliampere: $37.07 \mathrm{mAs}$, Voxel size: $0.25 \mathrm{~mm}$, Scanning time: 26.9 seconds, Field of view: $6 \mathrm{~cm}$ Height X $16 \mathrm{~cm}$ Diameter.

S-clean implants (Dentis Company, Korea) with diameters $(3.7 \mathrm{~mm})$ and length $(14 \mathrm{~mm})$. It has a tapered body design and Sandblasting with Large grit that facilitates the osteointegration process. The larger pitch of threaded design of dental implants to increase the implant primary stability. All implant sizes share the same internal hex.

After extra-oral disinfection of the surgical site. Patients were asked to rinse their mouths with Chlorohexidine HCL $1.25 \%$ mouthwash, and amoxicillin and clavulanic acid were administered orally 1 hour before procedure. All procedures were performed under local infiltration anesthesia 
[Articaine 4\%1:100000 epinephrine] was used. The extraction started using a periotome to clear the tissues around the root and to laxate the tooth. Then forceps were used to deliver the tooth out of its socket using gentle extraction movements to preserve buccal plate of bone. Then the socket was debrided and curated and irrigated with saline.

The integrity of the extraction socket was checked using a periodontal probe. If any dehiscence or fenestration defects were found, the case was excluded from the study. Drilling was started in a sequential manner and implant were placed bodily palatal in the undersized osteotomy to increase the primary stability followed by measurement of the jumping gap distance.

After that the torque wrench ratchet was attached to complete the seating of the implant into its final position, with implant placement sub-crestal at least $2 \mathrm{~mm}$ below cemento-enamel junction of adjacent tooth and 3-4 mm from FGM.

Then the primary stability was verified to be minimal $35 \mathrm{~N} / \mathrm{cm}$, the primary stability of each implant was measured using torque wrench ratchet, if the primary stability found to be less than $35 \mathrm{~N} /$ $\mathrm{cm}$. the socket seal provisional crown was fabricated instead of full anatomy temporary crown in order to prevent any kind of axial loading on the implant.

Acrylic crowns were prefabricated after removal of tooth on study cast that had been taken prior the surgery and after implant insertion the prefabricated acrylic crown picked up on abutment then abutment removed. Final contouring of prefabricated acrylic crown done by addition of flowable composite to achieve perfect emergence profile (concave shape interproximal to maintain papilla and labial to support labial gingiva).

Then, finishing of composite with finishing stones and polishing with wheel for achievement of smooth surface to encourage gingival formation and growth and to avoid bacterial and food debris accumulation. The relined provisional restoration was then disinfected.

All provisional crowns were made out of occlusion with a clearance of approximately $2 \mathrm{~mm}$; patients were instructed not to occlude on them and to use for esthetic purpose only, Finally, the provisional restoration was placed, all showed in figure (1).

For the first group (autogenous group); autogenous bone(ABG) graft taken by Auto chip maker (ACM bur) for augmentation of the gap between the implant and the labial plate of bone. Incision was made in the mucobuccal fold in the mandibular anterior region to expose the underlying bone. Autogenous bone graft was obtained from this region using the ACM BUR (speed $300 \mathrm{rpm}$, torque

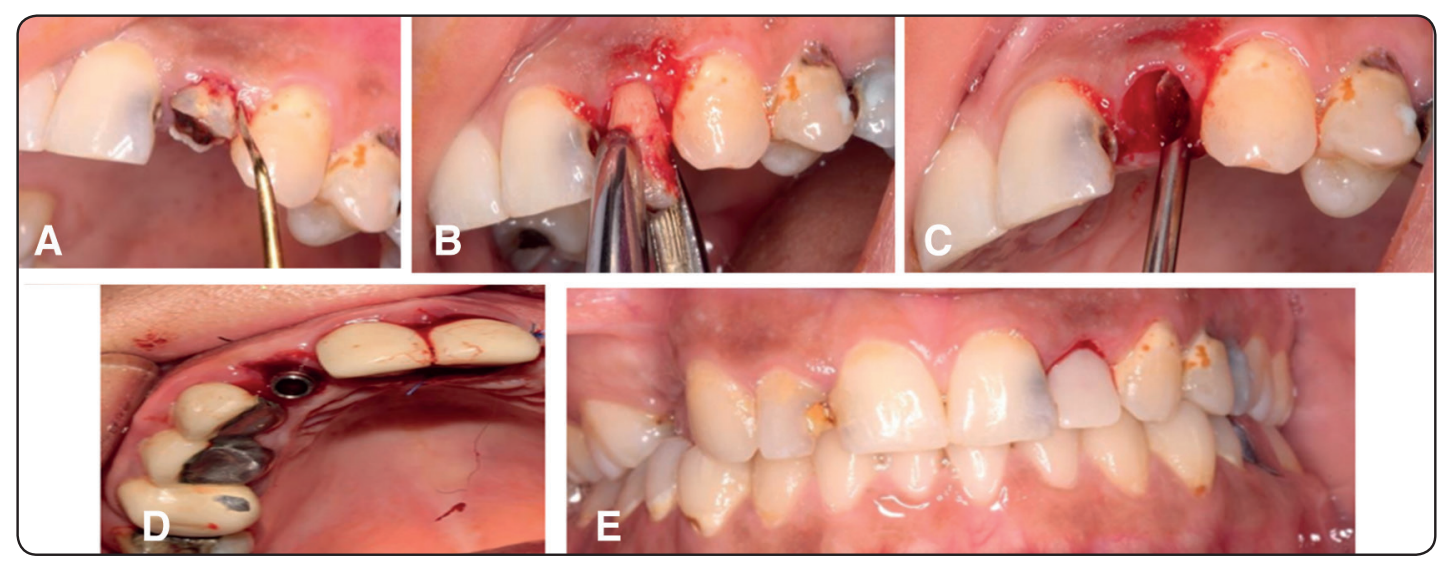

Fig. (1): Showing surgical procedures of immediate implant insertion (A) Periotome application. (B) Atraumatic extraction. (C) Socket debridement and curettage. (D) Bodily palatal position of implant.(E) Provisional restoration placement 
$50 \mathrm{Ncm}$ ). The cortical bone graft was collected and packed in the gap between the implant and the labial plate of bone . The flaps were sutured with 4-0 prolene sutures. In some cases we avoid additional surgery by drilling at low speed (300rpm) without irrigation to harvest bone chips from drill and fill the gap with it.

For the second group (xenograft group); using xenograft for augmentation of the gap between the implant and the labial plate of bone. We put a smooth and tall healing collar on an implant to allow the bone graft to be placed and packed against it by bone condenser up to the most coronal aspect of the FGM.

For the third group(nano-hydroxyapatite group); using nano-hydroxyapatite alloplastfor augmentation of the gap between the implant and the labial plate of bone.

All patients received post-operative antibiotic (amoxicillin and clavulanic acid) 1 gm every 12 hours orally for 3 days, NSAIDs orally for 3 days. Patients were instructed to follow oral hygiene measures and to use chlorohexidine $0.2 \%$ mouthwash for 2 weeks. All patients were clinically evaluated at 1 week, 2 weeks, 1 month and 6 months post operatively, all showed in figure (2).
Regarding horizontal bone level, showed in figure (3), on axial view drawing panoramic curve on the center of the teeth at the implant From panoramic view we select cross section passing through midline of implant. line is drawn passing through the center of implant $(3.7 \mathrm{~mm} / 2)$ and divided this line into two equal divisions connected with three points $(\mathrm{m}, \mathrm{m} 2, \mathrm{~m} 3)$. $\mathrm{m} 1$ indicated to point at apical part of implant $(14 \mathrm{~mm}), \mathrm{m} 2$ indicated to point at half of implant length $(7 \mathrm{~mm})$ and $\mathrm{m} 3$ indicated to cervical point at platform of implant. Another line is drawn from the labial margin of labial plate of bone perpendicular to the center line of the implant at $(\mathrm{m} 1, \mathrm{~m} 2$ and $\mathrm{m} 3)$ to record the distance between central line of implant to the labial margin of labial plate at three points $(\mathrm{m} 1, \mathrm{~m} 2, \mathrm{~m} 3)$. The average difference between the horizontal distances at 3 levels immediately after surgery and after 6 months represent the horizontal bone changes at different levels.

Regarding vertical bone level, showed in figure (4), on axial view drawing panoramic curve on the center of the teeth at the implant. From panoramic view, we select cross section passing through midline of implant. Then, line was drawn passing through the center of implant $(3.7 \mathrm{~mm} / 2)$. Another line was drawn passing through the implant platform perpendicular to the centerline of implant ( $\mathrm{T}$ line).
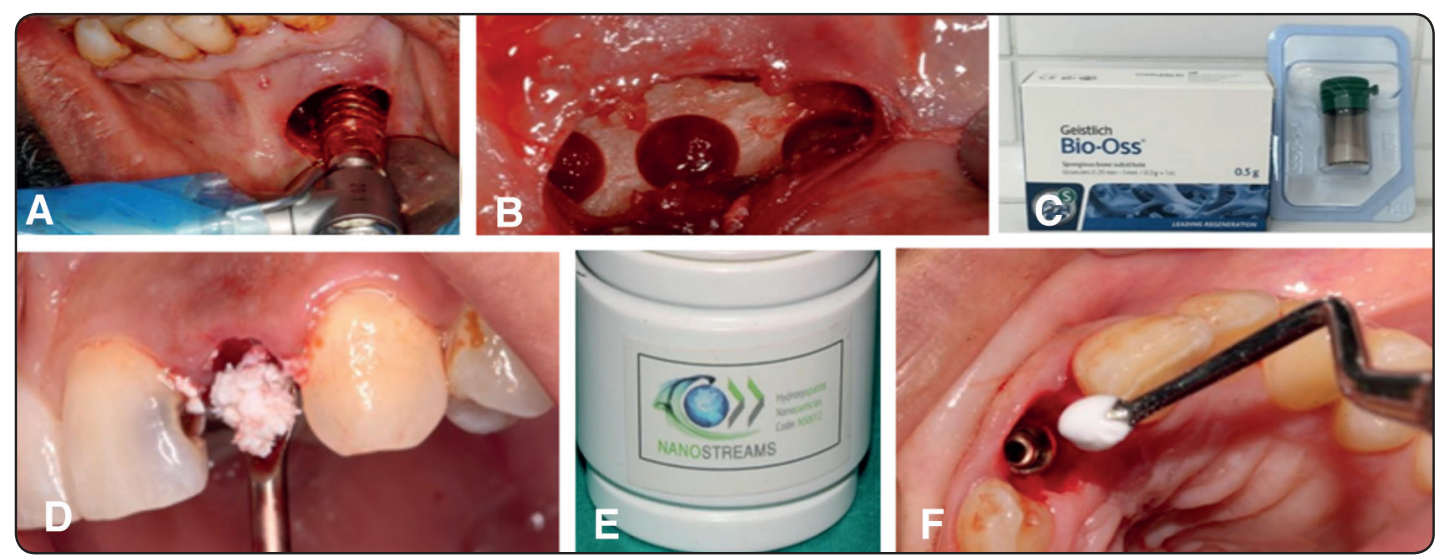

Fig (2): Showing surgical procedures of graft placement (A) harvesting of ABG using ACM. (B) Showing donor site after graft harvestation (symphysis) (C) showing xenograft particles. (D) showing application of xenograft. (E) showing nanohydroxyapatite graft. (F) showing application of nano bone. 
From the labial crest of bone a line was drawn perpendicular to the ( $\mathrm{T}$ line). The distance between the labial bone crest and the ( $\mathrm{T}$ line) was recorded immediately after surgery and 6 months postoperative and from palatal crest of bone at implant a line was drawn perpendicular to the ( $\mathrm{T}$ line) The distance between the palatal bone crest and the ( $\mathrm{T}$ line) was recorded immediately after surgery and 6 months post-operative The average difference between the vertical distances at immediately after surgery and after 6 months represent the vertical bone loss at different levels.

The Pink Esthetic Score (PES), showed in figure
(5), was based on seven variables: mesial papilla, distal papilla, soft- tissue level, soft tissue contour, alveolar process deficiency, soft-tissue color and texture. Each variable was assessed with a 2-1-0 score, with 2 being the best and 0 being the poorest score. The mesial and distal papilla were evaluated for completeness, incompleteness or absence. All other variables were assessed by comparison with a reference tooth,i.e. the corresponding tooth (anterior region) or a neighboring tooth premolar region. The highest possible score reflecting a perfect match of the peri-implant soft tissue with that of the reference tooth was 14.

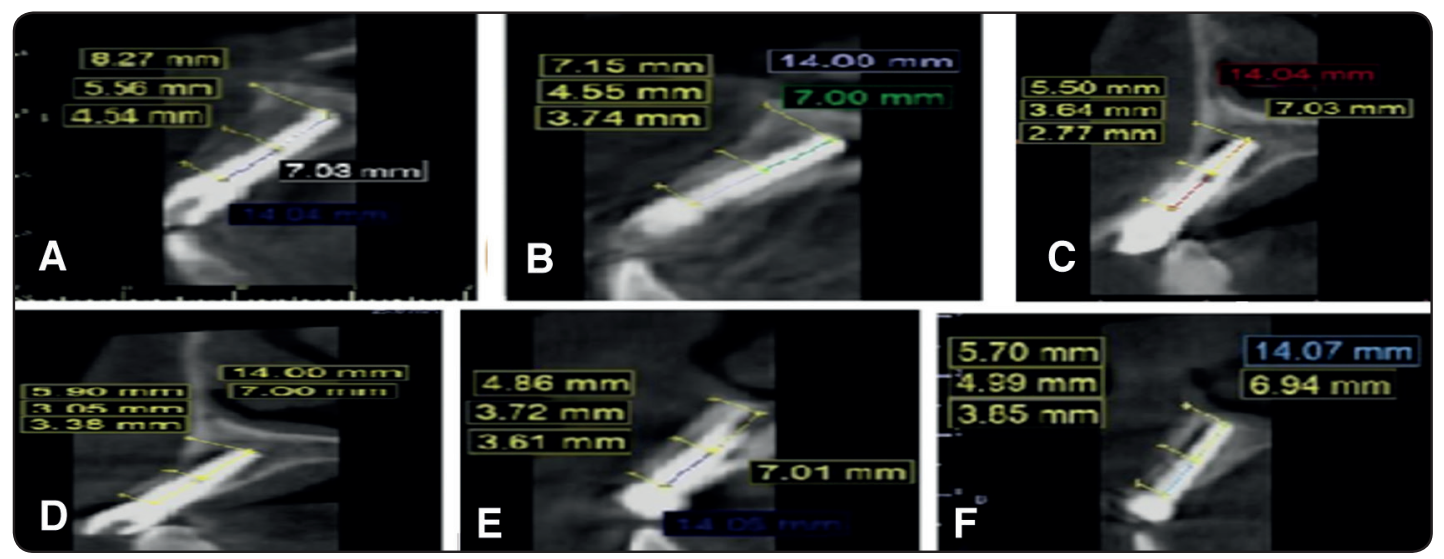

Fig. (3): Showing horizontal bone distance between (labial, palatal bony margin) and implant platform (T LINE). (A) immediately postoperative Autogenous group. (B) after 6 months Autogenous group.(C) immediately postoperative Xeno graft group. (D) after 6 months Xeno graft group.(E) immediately postoperative Nano-hydroxyapatite. (F) after 6 months Nanohydroxyapatite
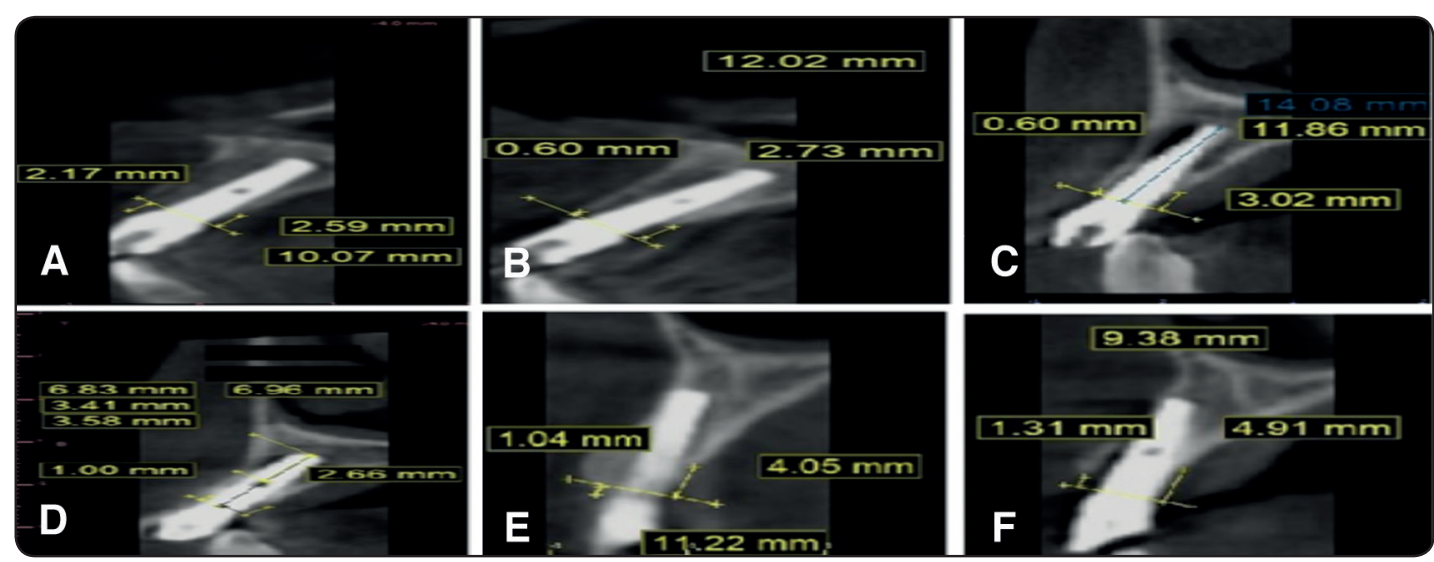

Fig. (4): Showing vertical bone distance between (labial, palatal bony margin) and implant platform (T LINE) immediately postoperative Autogenous group. (A) after 6 months Autogenous group. (B) immediately postoperative Xeno graft group. (C) after 6 months Xeno graft group. (D) immediately postoperative Nano-hydroxyapatite. (F) after 6 months Nanohydroxyapatite 


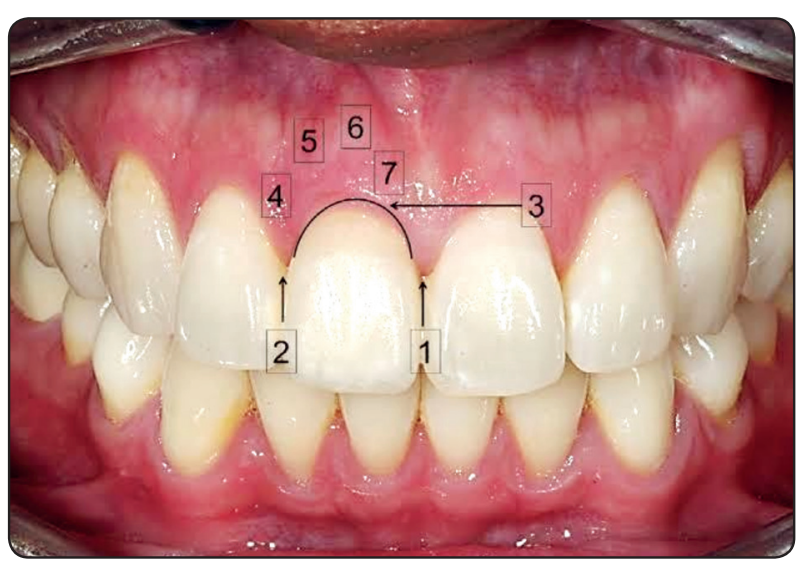

Fig. (5): Showing Criteria of PES evaluation

\section{RESULTS}

In the present study, thirty implants were placed in twenty patients (12 females and 8 males) requiring replacement of maxillary anterior teeth. The implants used had diameters of 3.7 and length of $14 \mathrm{~mm}$ in all groups. In the first group, 5 laterals and 4 centrals and 1 canine were extracted while in the second group 4 laterals and 3 centrals and 3 canines were extracted and in the third group, 6 laterals and 4 centrals were extracted.

Evaluating of overall horizontal bone loss of group, I (Autogenous Graft), group II (xenograft) and group III (nano-Hydroxyapatite), mean \pm standard deviation was $(0.6716 \pm 0.05378),(0.3806$ $\pm 0.02564)$ and $(0.2894 \pm 0.01394)$ respectively, all listed in table (1) and showed in figure (6). Using one-way analysis of variance test followed by Tukey`s post hoc test for multiple comparisons, there were insignificant difference between all groups, as value of probability level higher than 0.05 , listed in table (1).

Assessment of overall vertical bone Loss of group, I (Auto Graft), group II (Xeno Graft) and group III (Hydroxyapatite), mean \pm standard deviation was $(0.212 \pm 0.01576),(0.2335 \pm 0.01683)$ and $(0.2625 \pm 0.01902)$ respectively, all listed in table (1) and showed in figure (6). Using one-way analysis of variance test followed by Tukey`s post hoc test for multiple comparisons, there were significant difference between all groups, as value of probability level lower than 0.05 , listed in table (1).

After six months follow up, there were obvious advances the gingival and bony parameters in PES, using one-way analysis of variance (ANOVA) for each parameter of PES followed by Tukey's post hoc test for multiple comparisons, there was insignificant difference between three groups as value of probability level higher than 0.05 , as listed in table (2) and showed in figure (7).

TABLE (1): Comparison of Overall Horizontal and Vertical Bone Loss between Group I, II and III during Six Months Follow Up:

\begin{tabular}{|c|c|c|c|c|}
\hline \multirow{2}{*}{} & \multicolumn{3}{|c|}{ M \pm SD } & \multirow{2}{*}{ P-value } \\
\cline { 2 - 5 } & $\begin{array}{c}\text { Group I } \\
\text { (Auto Graft) }\end{array}$ & $\begin{array}{c}\text { Group II } \\
\text { (Xeno Graft) }\end{array}$ & $\begin{array}{c}\text { Group III } \\
\text { (Hydroxyapatite) }\end{array}$ & \multirow{2}{*}{$\mathbf{0 . 0 0}^{* *}$} \\
\hline Overall Horizontal Bone Loss & $0.6716 \pm 0.05378^{\mathrm{a}}$ & $0.3806 \pm 0.02564^{\mathrm{a}}$ & $0.2894 \pm 0.01394^{\mathrm{b}}$ & $\mathbf{0 . 0 0}^{* *}$ \\
\hline Overall Vertical Bone Loss & $0.212 \pm 0.1576^{\mathrm{a}}$ & $0.2335 \pm 0.1683^{\mathrm{a}}$ & $0.2625 \pm 0.1902^{\mathrm{b}}$ & \\
\hline
\end{tabular}

M: Mean, SD: Standard Deviation, P: Probability level

Means with same superscript letter in the same row were insignificant different

Means with different superscript letter in the same row were significant different

**significant difference 
TABLE (2): Comparison of PES scores between Group I, II and III during Six Months Follow Up:

\begin{tabular}{|c|c|c|c|c|c|c|c|}
\hline \multicolumn{7}{|c|}{ After Six Months } \\
\cline { 1 - 8 } & \multicolumn{2}{|c|}{$\begin{array}{c}\text { Group I (Auto } \\
\text { Graft) }\end{array}$} & $\begin{array}{c}\text { Group II (Xeno } \\
\text { Graft) }\end{array}$ & $\begin{array}{c}\text { Group III } \\
\text { (Hydroxyapatite) }\end{array}$ & \multirow{2}{*}{ P-value } \\
\cline { 2 - 8 } & M & SD & M & SD & M & SD & \\
\hline Mesial papilla & $1.62 \mathrm{a}$ & 0.08 & $1.98 \mathrm{~b}$ & 0.07 & $1.48 \mathrm{c}$ & 0.02 & \multirow{0}{*}{$\mathbf{0 . 0 0 * *}$} \\
\hline Distal papilla & $1.7 \mathrm{a}$ & 0.034 & $1.84 \mathrm{~b}$ & 0.05 & $1.51 \mathrm{c}$ & 0.011 & $\mathbf{0 . 0 0 * *}$ \\
\hline Level of soft-tissue margin & $1.94 \mathrm{a}$ & 0.021 & $1.2 \mathrm{~b}$ & 0.033 & $1.98 \mathrm{a}$ & 0.017 & $\mathbf{0 . 0 0 * *}$ \\
\hline Soft-tissue contour & $1.12 \mathrm{a}$ & 0.084 & $1.51 \mathrm{~b}$ & 0.047 & $1.27 \mathrm{c}$ & 0.091 & $\mathbf{0 . 0 0 * *}$ \\
\hline Alveolar process & $1.05 \mathrm{a}$ & 0.027 & $1.76 \mathrm{~b}$ & 0.061 & $1.04 \mathrm{a}$ & 0.025 & $\mathbf{0 . 0 0 * *}$ \\
\hline Soft-tissue color & $1.57 \mathrm{a}$ & 0.036 & $1.23 \mathrm{~b}$ & 0.024 & $1.71 \mathrm{a}$ & 0.052 & $\mathbf{0 . 0 0 * *}$ \\
\hline Soft-tissue texture & $1.14 \mathrm{a}$ & 0.081 & $0.87 \mathrm{~b}$ & 0.033 & $1.27 \mathrm{c}$ & 0.073 & $\mathbf{0 . 0 0 * *}$ \\
\hline Overall & $10.14 \mathrm{a}$ & 0.363 & $10.39 \mathrm{a}$ & 0.318 & $10.26 \mathrm{a}$ & 0.289 & $\mathbf{0 . 2 4 5 0}$ \\
\hline
\end{tabular}

M: Mean, SD: Standard Deviation, P: Probability level

Means with same superscript letter in the same row were insignificant different

Means with different superscript letter in the same row were significant different

*insignificant difference

**significant difference

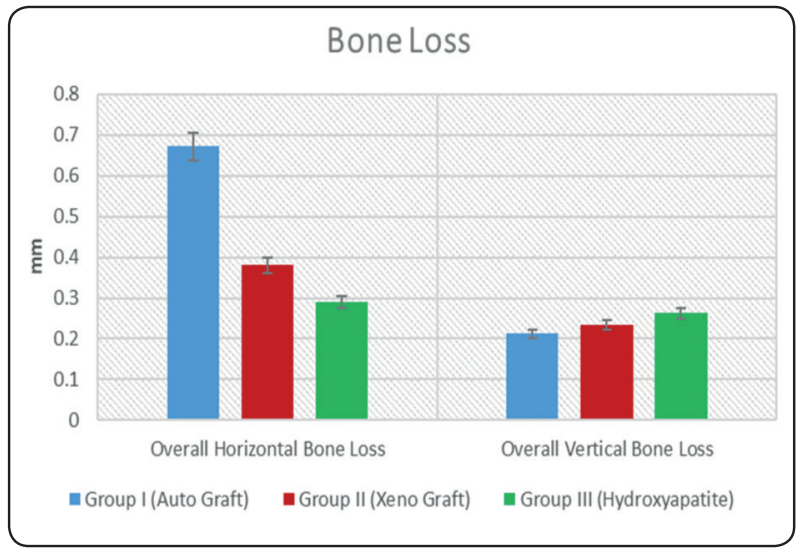

Fig. (6): Bar Chart Overall Horizontal and Vertical Bone Loss between Group I, II and III after Six Months Follow Up

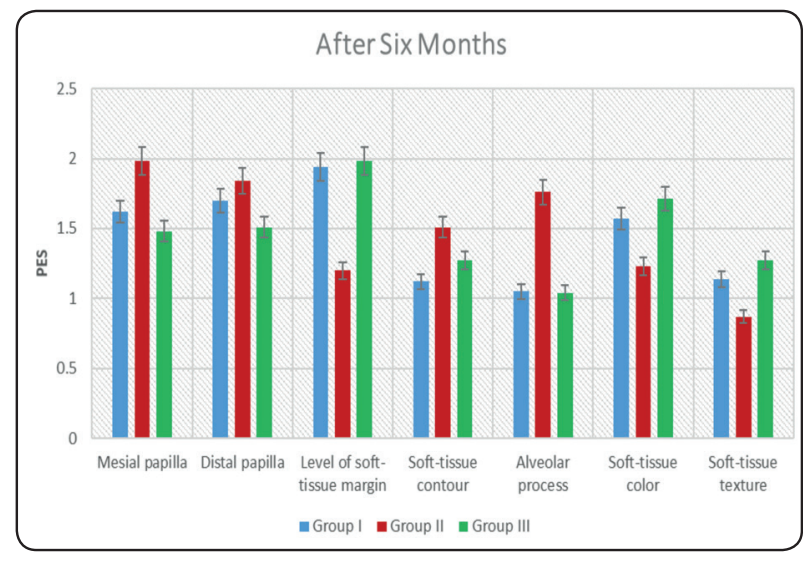

Fig. (7): Bar Chart PES scores between Group I, II and III after Six Months Follow Up 


\section{DISCUSSION}

In the present study all the implants were inserted with flapless technique because it had advantages of less recession of the peri-implant area, less morbidity for the patients, simple surgery and this comes in agreement with other researches who preferred flapless technique because there was only three sources of blood supply to the facial plate of bone ( the periodontal ligament, the labial periosteum, and the endosseous marrow) Once any tooth is extracted, the ligament blood supply was absent $^{(12)}$.

The procedure of drilling was performed at a maximum rotation speed of $1100 \mathrm{rpm}$. As The bone drilling at $1100 \mathrm{rpm}$ in dense bone generates less heat than at slower speeds because it reduces the time of drilling. But in two cases of our study we found clinically soft bone palatal so we decrease the speed of drilling to (300 rpm) without irrigation which led us to harvest autologous bone without the need for additional surgery and proper path of drill in bodily palatal position. And that comes in agreement with other researches which concluded that bone chips obtained from drilling at low speed had higher quality than bone obtained from conventional drilling ${ }^{(13)}$.

In the present study we used two-piece implant that had tapered body design which mimic more the natural root shape compared with the cylindrical implant design because the size of its diameter decrease towards the apical region which leads distribute forces into surrounding bone, created lateral compression of bone, increase the primary stability in low-density bone and less risk of labial perforation and this comes in agreement with other researches. And the most important thing about tapered design is to accommodate the labial concavity of anterior maxilla ${ }^{(14)}$.

There was usually a gap between labial margin of labial plates \& implant called "jumping gap", the gap may be remained with bone graft if it more than $2 \mathrm{~mm}$ or remain without bone graft if it less than $2 \mathrm{~mm}$ which showed spontaneous healing without graft. Autogenous, xenograft, and synthetic bone substitutes and/or materials can be used in the gap to graft the bone and tissue zones. The graft material served as a scaffold to maintain hard- and soft-tissue volume as well as blood clot for initial healing. Bone grafts eliminated post-surgical buccal bone resorption. The principle standard material for grafting protocol was autogenous graft due to no risk of failure. In some cases of first group we use ACM bur to harvest autogenous bone chips to use it to fill the gap, and this more morbidity to patient because more needed surgical site was required (chin and osteotomy in anterior maxilla), another potential location for postoperative pain and complications. Advantages of ACM bur was keep well viability of bone and while it rotated not compromised the soft tissue. And mostly considered harvest cortical bone chips not cortico-cancellous chips. Advantages of cortical bone over cortico-cancellous was cortical bone more morphogenic and lead to earlier bone union than cortico-cancellous bone grafting and this comes in agreement with other researches. So in some cases to avoid additional surgery we drilling at low speed (300 rpm) without irrigation to collect bone chips from drill and fill the gap with it. ACM bur was usually used more than Trephine or alveolar bone scraper because bone blocks from trephine must crushed properly. If not, it may be cause infection of graft and also compromised the viability of bone. We not need to harvest autogenous bone graft as space filling material in jumping gap according to our result there were insignificant difference between all groups ${ }^{(15)}$.

Using of an immediately placed provisional restoration that mirrors the cervical contours of the extracted tooth at the time of implant placement may provide a platform to promote peri-implant soft tissue healing. In the present study we used screw-retained provisional restorations which prefabricated acrylic crown on study cast prior 
surgery and after implant insertion the prefabricated acrylic crown picked up on abutment then abutment removed and build up the emergence by flowable composite (slightly concave emergence profile). The relined provisional restoration was then disinfected. This technique was easier, cheaper and faster than other techniques. This technique comes in agreement with recent researches ${ }^{(16)}$.

In the present study horizontal reduction in the labial bone plate following flapless immediate implant placement in the anterior maxilla was evaluated on CBCT scans and the effect of labial gap filling with slow resorption bone material on the reduction of labial bone height was evaluated. Our results showed that after 6 months of healing, the width of labial bone wall undergo a mean reduction of $(0.671) \mathrm{mm},(0.380) \mathrm{mm}$ and $(0.289) \mathrm{mm}$ in the first, second and third groups, respectively which it was statistically not significant $\mathrm{p}=(0.057)$. It was reported after immediate implant placement and the jumping gap filled with an autologous bone graft material approximately, $\mathrm{X}$ ray investigation showed only slight marginal bone reduction of $(0.13) \mathrm{mm}$ mesially and (0.19) mm distally ${ }^{(17)}$.

It was also showed after immediate implant with immediate provisionalization the mean bone resorption of (0.5) mm after one year follow up. this came in agreement with our study due to he used temporization which have major role in outcomes. Our results showed that after 6 months of healing, the height of labial bone wall undergoes a mean reduction of $(0.212) \mathrm{mm},(0.233) \mathrm{mm}$ and $(0.262)$ $\mathrm{mm}$ in first, second and third groups respectively ${ }^{(18)}$.

It was reported that after immediate implantation in the anterior maxilla with filling the gap with bovine graft (Bio-oss) the mean horizontal bone loss of buccal bone about $(0.5) \mathrm{mm}$ and our result showed (0.3) $\mathrm{mm}$ so we considered that immediate temporization played major role in raised outcomes ${ }^{(19)}$.

It was studied the bone volume changes after immediate implant placement with simultaneous grafting by an organic bovine bone with flapelevation technique in non-molar region and reported a mean reduction in height (1.03) $\mathrm{mm} \&$ mean reduction in width about (1.09) $\mathrm{mm}$ and this result was higher than our result $(0.262) \mathrm{mm},(0.380) \mathrm{mm}$ because we used flapless technique which make major role in immediate implant placement ${ }^{(20)}$.

Another purpose of this study was to evaluate the bone grafts role on soft tissue by using pink esthetic score to evaluate those changes after 6-month follow up. The first index called papilla fill index. It only concentrated on evaluation of interproximal papilla only but pink esthetic score which evaluate mesial papilla and distal papilla and tissue height, position, color and texture. The total mean PES in our study was (10.14), (10.39) and (10.58) in first, second and third groups, respectively. The mean PES of this presented study was closely matching with the result demonstrated by other researches after soft tissue preservation on twenty-two immediate implant which were 12 (mean PES) ${ }^{(21)}$.

Also, it was studied forty single-unit implants placed in the anterior maxilla and restored with screw-retained or cement-retained crowns in function for a minimum of one year and reported the mean PES was (10.73) and (10.41) in the test and control group respectively. Finally, all mean of PES was closely matched with result of this study ${ }^{(22)}$.

\section{CONCLUSION}

Immediate implant with immediate temporization using graft materials to augment jumping gap was successful treatment modality for rehabilitation of hopeless teeth in the esthetic zone. The type of graft materials doesn't significantly affect on the outcome (horizontal and vertical bone loss) following implant installation with immediate temporization in the esthetic zone. 


\section{REFERENCE}

1. Abushahba F, Renvert S, Polyzois I, Claffey N. Effect of grafting materials on osseointegration of dental implants surrounded by circumferential bone defects. An experimental study in the dog. Clin Oral Implants Res. 2008;19(4):329-334. doi:10.1111/j.16000501.2007.01455.x

2. Singh M, Kumar L, Anwar M, Chand P. Immediate dental implant placement with immediate loading following extraction of natural teeth. Natl J Maxillofac Surg. 2015;6(2):252-255. doi:10.4103/0975-5950.183864

3. Mehta H, Shah S. Management of Buccal Gap and Resorption of Buccal Plate in Immediate Implant Placement: A Clinical Case Report. J Int Oral Health. 2015; 7(Suppl 1):72-75.

4. Tarnow, Dennis \& Chu, Stephen. (2011). Human histologic verification of Osseo-integration of an immediate implant placed into a fresh extraction socket with excessive gap distance without primary flap closure, graft, or membrane: a case report. The International journal of periodontics \& restorative dentistry.2011.

5. Antunes AA, Grossi-Oliveira GA, Martins-Neto EC, Almeida AL, Salata LA. Treatment of circumferential defects with osseoconductive xenografts of different porosities: a histological, histometric, resonance frequency analysis, and micro-CT study in dogs. Clin Implant Dent Relat Res. 2015;17 Suppl 1:e202-e220. doi:10.1111/cid.12181.

6. Noelken R, Moergel M, Kunkel M, Wagner W. Immediate and flapless implant insertion and provisionalization using autogenous bone grafts in the esthetic zone: 5-year results. Clin Oral Implants Res. 2018;29(3):320-327. doi:10.1111/ clr.13119

7. Ebenezer V, Balakrishnan K, Sargunar R. B, Senthilnadhan. Nano Hdroxyapatite Particulate Graft in Immediate Implant Placement - A Review Of 10 Cases. Biomed Pharmacol J 2015;8(October Spl Edition)

8. Attia MS, Mohammed HM, Attia MG, Hamid MAAE, Shoeriabah EA. Histological and histomorphometric evaluation of hydroxyapatite-based biomaterials in surgically created defects around implants in dogs. J Periodontol. 2019;90(3):281-287. doi:10.1002/JPER.17-0469

9. Van Nimwegen WG, Goené RJ, Van Daelen AC, Stellingsma K, Raghoebar GM, Meijer HJ. Immediate implant placement and provisionalisation in the aesthetic zone. J Oral Rehabil. 2016;43(10):745-752. doi:10.1111/ joor. 12420

10. Arora H, Khzam N, Roberts D, Bruce WL, Ivanovski S. Immediate implant placement and restoration in the anterior maxilla: Tissue dimensional changes after 2-5 year follow up. Clin Implant Dent Relat Res. 2017;19(4):694702. doi:10.1111/cid.12487

11. Elshenawy H, Aly W, Salah N, Nasry S, Anter E, Ekram K. Influence of Small, Midi, Medium and Large Fields of View on Accuracy of Linear Measurements in CBCT Imaging: Diagnostic Accuracy Study. Open Access Maced J Med Sci. 2019;7(6):1037-1041. Published 2019 Mar 29. doi:10.3889/oamjms.2019.232

12. Tarnow DP, Chu SJ, Salama MA, et al. Flapless postextraction socket implant placement in the esthetic zone: part 1. The effect of bone grafting and/or provisional restoration on facial-palatal ridge dimensional change-a retrospective cohort study. Int J Periodontics Restorative Dent. 2014;34(3):323-331.

13. Canullo L, Rasperini G. Preservation of peri-implant soft and hard tissues using platform switching of implants placed in immediate extraction sockets: a proof-of-concept study with 12- to 36-month follow-up. Int J Oral Maxillofac Implants. 2007;22(6):995-1000.

14. George F. and Mahmoud Abdel-Aziz Ibrahim Khalil. "Compression Between Cylindrical and tapered implants in Delayed Immediate placement .2016.

15. Kim DH, Kim KI, Yoon S, et al. Dental Hetero-Graft Materials with Nano Hydroxyapatite Surface Treatment. J Nanosci Nanotechnol. 2015;15(10):7942-7949. doi:10.1166/ jnn.2015.11197.

16. Scala R, Ghensi P, Cucchi A, Pistoia E. Postextraction implant placement with immediate provisionalisation and finalisation, using a simplified technique: technical notes and a case report. Open Dent J. 2012;6:164-169. doi:10.2174/1874210601206010164

17. Schwartz-Arad D, Laviv A, Levin L. Survival of immediately provisionalized dental implants placed immediately into fresh extraction sockets. J Periodontol. 2007;78(2):219-223. doi:10.1902/jop.2007.060288

18. Bonnet F, Karouni M, Antoun H. Esthetic evaluation of peri-implant soft tissue of immediate single-implant placement and provisionalization in the anterior maxilla. Int $\mathrm{J}$ Esthet Dent. 2018;13(3):378-392. 
19. Botticelli, D.; Berglundh, T. and Lindhe, J. (2004): Hardtissue alterations following immediate implant placement in extraction sites. J. Clin. Periodontal; 31(10):820-828.

20. Jordana F, Le Visage C, Weiss P. Substituts osseux [Bone substitutes]. Med Sci (Paris). 2017;33(1):60-65. doi:10.1051/medsci/20173301010

21. Cosyn J,Hooghe N, De Bruyn H.A systematic review on the frequency of advanced recession following single immediate implant treatment. J Clin Periodontol. 2012;39(6):582589. doi:10.1111/j.1600-051X.2012.01888.x

22. Cutrim ES, Peruzzo DC, Benatti B. Evaluation of soft tissues around single tooth implants in the anterior maxilla restored with cemented and screw-retained crowns. J Oral Implantol. 2012;38(6):700-705. doi:10.1563/AAID-JOID-11-00125 